\title{
TOP CITED PAPERS IN INTERNATIONAL PSYCHOGERIATRICS: 5. A CONTROLLED STUDY OF REPETITIVE TRANSCRANIAL MAGNETIC STIMULATION AS A TREATMENT OF DEPRESSION IN THE ELDERLY
}

\section{Reflection}

We were delighted, but surprised, by the publication success of our first pilot trial on the efficacy of repetitive transcranial magnetic stimulation (rTMS) to treat late life depression (LLD) (Manes et al., 2001). This preliminary study had major limitations - for example, the small sample size and the use of both a non-therapeutic dose of rTMS and a defective method of sham stimulation. However, it addressed an important clinical problem for which we still do not have a satisfactory solution: how do we treat LLD that has been resistant to the usual therapeutic approaches in a safe and efficient way?

In order to give a tentative response to this question we need to describe briefly the characteristics of LLD, the available evidence regarding the efficacy of common antidepressants in this condition, and the role that coexisting cerebrovascular disease plays in treatment response.

\section{Characteristics of LLD}

Late life depression occurs in up to $5 \%$ of community-dwelling elderly and its frequency is higher in medical settings (Mulsant and Ganguli, 1999). LLD aggravates comorbid medical conditions and increases disability (Lebowitz et al., 1997; Charney et al., 2003). In addition, LLD is associated with cognitive impairment, affecting information processing speed, executive function, and working memory (Nebes et al., 2001; 2002; Butters et al. 2000; 2004).

It has been estimated that about half of LLD patients have MRI evidence of subcortical ischemic damage affecting deep white matter pathways, thalamus and basal ganglia (Krishnan et al., 2004). These neuroimaging findings have been the basis for proposing a distinct subtype of LLD: vascular depression (VaD) (Alexopoulos et al., 1997; Krishnan et al., 1997).

Subcortical hyperintensities found in $\mathrm{VaD}$ correlate with psychomotor slowing (Hickie et al., 1995; Simpson et al., 1997; Simpson et al., 2000), poor outcomes of depression (Hickie et al., 1995;

First published online 10 July 2009
Simpson et al., 1997; 2000) functional impairment (Steffens et al., 2002) and cognitive deficits (Lesser et al., 1996; Salloway et al., 1996; Simpson et al., 1997). Furthermore, investigators at Duke University have shown that deep white matter hyperintensities (WMH) are independently associated with mortality in depressed older patients (Levy et al., 2003).

\section{Efficacy of antidepressants in LLD}

Roose and Schatzberg (2005) analyzed the available evidence on the efficacy of antidepressants for treatment of LLD. The clinical trials examined in this analysis did not select for patients with $\mathrm{VaD}$, although many subjects would likely meet criteria for this diagnosis. Despite some discordant findings - two out of five methodologically rigorous studies did not find that antidepressants were more effective than placebo in this population (Roose et al., 2004; Schatzberg and Roose, 2006) - the authors concluded that antidepressants are effective in the treatment of LLD. However, they also pointed out that as many as $50 \%$ of patients do not achieve symptomatic remission.

\section{Do subcortical ischemic lesions influence treatment response in LLD?}

Currently, there is some controversy with regard to this issue. Previous studies of $\mathrm{VaD}$ indicate that this form of depression may be more difficult to treat over time. For instance, Alexopoulos et al. have reported that, when compared with other older depressed patients, individuals with $\mathrm{VaD}$ have slower recovery, only partial response to treatment with antidepressants, executive deficits and a more chronic course (Hickie et al., 1995; Lesser et al., 1996; Alexopoulos et al., 1997) Furthermore, in a longitudinal MRI study, depressed elders who did not achieve or sustain remission had a significantly greater increase in WMH volume over time than did the group that did achieve and sustain remission (Taylor et al., 2003). On the other hand, Salloway et al. (2002) reported that among 39 elderly depressed patients treated with sertraline and 111 depressed patients treated with citalopram, there was no relationship between treatment response 
and severity of WMH. In addition, a more recent study of very old depressed patients did not find a significant correlation between quantitative measures of white matter lesions and response to antidepressant treatment with citalopram (Sneed et al., 2007).

However, we can hypothesize that, rather than the total burden of lesions, the location of ischemic damage plays a significant role in the clinical response to different antidepressants. For example, among 13 older depressed patients receiving citalopram, having decreased fractional anisotropy of ventral frontal white matter tracts was associated with a lower rate of remission (Alexopoulos et al., 2002).

In summary, a significant proportion of patients with LLD will have a limited response to antidepressants. Although it is not clear if the presence of subcortical ischemic damage is a significant contributor to treatment resistance to usual antidepressants, it is extremely important to develop therapeutic alternatives to increase response and remission rates among patients with different forms of LLD.

\section{Is rTMS a safe and effective therapeutic option for this group of depressive disorders?}

To answer this question, we need, first, to examine the current evidence on the magnitude and clinical relevance of rTMS antidepressant effect. Secondly, we need to demonstrate the efficacy of rTMS to treat LLD and, particularly, VaD.

With regard to the first issue, Loo and Mitchell (2005) recently reviewed seven meta-analyses of sham-controlled rTMS studies in major depression. Varying inclusion criteria produced a range in the number of studies covered in the seven analyses of five to 16 . Only one meta-analysis, though, concluded that significant evidence for the efficacy of rTMS was lacking (Couturier, 2005). On the other hand, five studies found clear evidence of efficacy. Although there is consensus that TMS has statistically significant antidepressant effects, a more important question is whether these effects are clinically relevant. The meta-analyses above reported, on average, an effect size of Cohen's D of about 0.65 , which is a moderate effect, in the same range as the effects of antidepressant medications.

It is important to emphasize that, during the past few years, rTMS trials have moved in the direction of administering longer duration protocols with higher intensities and greater total cumulative doses of magnetic pulses (Janicak, et al., 2002; Gershon et al., 2003; Grunhaus et al., 2003). There has been consistent progress in the implementation of the technique and in the design of appropriate sham stimulation methods. In addition, small single site studies have given way to more powerful multicenter projects. For instance, the results of the first industry-sponsored multicenter trial (Neuronetics) were recently available for analysis (O'Reardon et al., 2007). In a double blind, multisite study, 301 medication-free patients with major depression, who had not benefited from prior treatment, were randomized to active TMS $(\mathrm{n}=155)$ or sham TMS $(n=146)$ conditions. The primary outcome was the symptom score change as assessed at week 4 with the Montgomery-Åsberg Depression Rating Scale (MADRS). Secondary outcomes included changes on the 17- and 24-item Hamilton Depression Rating Scale (HDRS), and response and remission rates with the MADRS and HDRS. Active TMS was significantly superior to sham TMS on the MADRS at week 4 (with a post-hoc correction for inequality in symptom severity between groups at baseline), as well as on the HDRS-17 and HDRS24 scales at weeks 4 and 6 . Response rates were significantly higher with active TMS on all three scales at weeks 4 and 6 .

Remission rates were approximately two-fold higher with active TMS at week 6 on all scales. The effect size of the difference between HDRS17 scores among the 301 subjects was 0.55 (i.e. in the moderate range). Furthermore, secondary analysis of the group of patients who were less treatment resistant (i.e. those patients who have failed only one adequate pharmacological trial) showed an effect size of 0.85 , significantly greater than the one usually observed following treatment with usual antidepressants. In addition, rTMS was well tolerated, with a low dropout rate for adverse events $(4.5 \%)$ that were generally mild and limited to transient scalp discomfort or pain (O'Reardon et al., 2007).

Regarding rTMS efficacy among patients with LLD, two previous small controlled studies failed to show a significant effect of active rTMS (Manes et al., 2001; Mosimann et al., 2004). The first of them was conducted by our group and is the one published in this journal in 2001. The study had the major drawbacks noted above. In the second one (Mosimann et al., 2004), high frequency left prefrontal rTMS was shown to have no specific effect on depression. In addition to the small sample size, this study had several methodological limitations such as the inclusion of several patients with bipolar disorder or early onset recurrent depressive disorder in the active but not the sham treatment group, an inadequate sham procedure, and a relatively low stimulation intensity $(100 \% \mathrm{MT})$ for this age group. Furthermore, they used an empirical algorithm to localize the 
stimulation site that does not guarantee that they were actually stimulating the dorsolateral prefrontal cortex (DLPC) (Mosimann et al., 2004).

A small, open study has examined response to rTMS in treatment resistant $\mathrm{VaD}$ (Fabre et al., 2004). The results demonstrated that five of 11 patients were treatment responders with a drop in HDRS scores between 10 and 14 points. The mean HDRS score in the responder group decreased from $24 \pm 5.9$ to $12.6 \pm 5.5$ while the six nonresponders had pretreatment HDRS scores of $24.5 \pm 7.2$ versus post treatment $24.8 \pm 5.9$.

We have recently completed a NIH-sponsored project on the efficacy of rTMS to treat depressive disorders associated with cerebrovascular disease. We conducted two independent experiments comparing active left DLPC rTMS with sham stimulation. In the first one, we delivered a total cumulative dose (TCD) of 12,000 magnetic pulses while in the second one the TCD was increased to 18,000 magnetic pulses. To our knowledge, these are the first randomized controlled trials of the efficacy of rTMS to treat $\mathrm{VaD}$ and the largest trial in LLD.

In Experiment 1, the sham group showed a $13.6 \%$ decrease in HDRS-17 scores compared to $33.1 \%$ decrease in the TCD-12K group $(\mathrm{p}=0.04)$. Response rates were $6.7 \%$ in the sham group and $33.3 \%$ in the active group $(\mathrm{p}=0.08)$ and remission rates were $6.7 \%$ in the sham and $13.3 \%$ in the active group $(p=0.50)$. In Experiment 2 , the sham group showed a $17.5 \%$ decrease in HDRS-17 scores compared with a $42.4 \%$ decrease observed in the TCD-18K group $(\mathrm{p}=0.0001)$. Response rates were $6.9 \%$ in the sham group and $39.4 \%$ in the active group $(\mathrm{p}=0.003)$, and remission rates were $3.5 \%$ in the sham group and $27.3 \%$ in the active group $(p=0.01)$. The effect size of the difference between HDRS scores was 0.89 in this group of $\mathrm{VaD}$ patients.

Age had a significant impact on the antidepressant response to rTMS. Among older patients (65 years or more), active rTMS produced significantly greater reductions of HRSD-17 scores than sham stimulation only in the group of patients who received the higher rTMS dose (TCD-18K).

\section{Commentary}

As we are all well aware, depression in older people remains one of the great challenges in our field. Prevalence of major depression in older people is usually cited as around $3 \%$, but minor depressive symptoms are up to fivefold more common, may
Greater antidepressant response to rTMS was significantly associated with higher frontal grey matter volumes. rTMS was safe, well tolerated, and not associated with cognitive deficits. We need to emphasize that a higher dose of left DLPC rTMS was associated with greater antidepressant efficacy. It is highly probable that we have not yet found an optimal TCD of rTMS to achieve maximal response and remission rates in this population.

In summary, there is consistent preliminary evidence suggesting that rTMS is a safe and effective antidepressant intervention among patients with VaD. However, future studies should examine several important issues. The first issue relates to the selection of those rTMS stimulation parameters associated with the higher response and remission rates that can be achieved by rTMS among this group of patients. A second issue is the need to determine whether $\mathrm{VaD}$ represents a subgroup of LLD that shows a greater response to rTMS or if, after optimization of the stimulation parameters, LLD patients without evidence of ischemic damage will show similar response to rTMS as VaD patients. Finally, if VaD proves to be more responsive to rTMS than other forms of LLD, we should investigate the underlying mechanism of this phenomenon.

Further studies should rely on a multicenter approach in order to enroll a larger sample of LLD patients that will allow more meaningful statistical inferences to be made. In addition, it is advisable to increase the dose of rTMS significantly, incorporate state of the art techniques to localize the stimulation site, adjust the rTMS dose to the degree of prefrontal atrophy, and optimize the sham stimulation condition.

Needless to say, LLD is a major health concern for which we still do not have optimal therapeutic options. rTMS constitutes a reasonable alternative considering both efficacy and safety issues. In our opinion, the available evidence clearly supports the need for further research in this field.

\section{RiCARDo E. JORGE AND RoBERT G. RobINSON \\ Department of Psychiatry, Roy J and Lucille A. Carver \\ College of Medicine, University of Iowa, U.S.A. \\ Email: Ricardo-jorge@uiowa.edu}

be as disabling as major depression and carry a poor prognosis. For example, Chuan et al. (2008) found that subsyndromal depression had similar adverse consequences on mental, physical and functional status as syndromal cases. In a 12 year follow-up, Andreescu et al. (2008) showed that minor depressive symptoms were associated with 
future increases in symptoms, especially if subjects were female or had functional disability. Depressive symptoms were also more likely to persist over time in those with greater medical burden. Similarly, Lyness et al. (2009) showed that older people with minor and subsyndromal depression had a sevenfold risk of developing major depression over the subsequent year.

Despite its high prevalence, current treatments for depression in late life are arguably still very limited. The development of new and effective treatments for depression is especially challenging as, despite much knowledge about risk factors for depression, etiology remains essentially unknown and many of the established risk factors are not easy to modify, if they can be modified at all. The translation of treatments from younger adults to older adults cannot be automatically assumed, yet the evidence base supporting drug prescribing for affective disorders in old people is limited. For example, a recent survey of Old Age Psychiatrists found that although prescription rates for mood stabilizing drugs was high, over half of respondents indicated that lack of scientific evidence was a major concern when prescribing treatments for mood disorders in older people (Ephraim and Prettyman, 2009). Although antidepressants are effective in older people (Raskin et al., 2008), even novel dual acting agents such as duloxetine show nonresponse rates (as defined by greater than a 50\% reduction in HDRS scores) as high as $60 \%$ at 8 weeks (Raskin et al., 2008). Better treatments are desperately needed, especially for those who do not respond to standard antidepressant treatments. Electroconvulsive therapy is widely accepted as an efficacious treatment for late life depression, although of course there are risks (albeit small) in older people because of the need for anesthesia. There is also a powerful anti-ECT lobby which undoubtedly has had an influence in the varied prescribing rates for ECT found both over time and between different countries. The search for a safe and effective alternative to ECT is therefore of great importance.

The top-cited paper under discussion (Manes et al., 2001) is a good example of a pioneering study in this regard and one that has had significant impact in the field. The authors applied rapid TMS (rTMS) (Jorge et al., 2008) to the left dorsal lateral cortex in depressed subjects finding no significant benefits (compared to sham treatment) at seven days' follow-up. Intriguingly, they found that responders to treatment had significantly greater frontal lobe volume than nonresponders, suggesting that there may be an important interaction between structural brain abnormalities (which have been well identified in depression) and response to treatment, as others have previously shown (O'Brien et al., 1998; Almeida et al., 2003). As well as providing an excellent summary of the field and commentary in their reflection piece, Jorge and Robinson also describe two new studies of TMS of left dorsal atrophy frontal cortex compared with sham stimulation. Although full details, such as subject details and numbers of subjects, are described elsewhere (Jorge et al., 2008) the authors report benefits in terms of decreased HDRS scores (but not response rates or admission rates) in Experiment 1 and highly significant benefits of TMS in terms of reduced HDRS scores, response rates and remission rates in subjects with depression associated with cerebrovascular disease. Such a "vascular depression" has been categorized as a subtype of depression in older people which may have a specific clinical and cognitive profile and implications with regard to treatment. However, uncertainties about the relevance of vascular changes and how such people should be identified remains. As Almeida (2008) points out, vascular risk factors and cerebrovascular disease are so common in older people that if these alone are used to categorize subjects, almost anyone who develops depression after the age of 65 may be deemed to have a vascular depression. However, Jorge and colleagues are to be congratulated for undertaking one of the few therapeutic studies in the area, since it is only by further research in this area that the true significance of vascular factors and their implications for treatment can be determined. Their new studies directly result from their original pioneering TMS top-cited paper.

Intriguingly, in their recent studies, Jorge and colleagues have found that greater antidepressant response to rTMS is significantly associated with higher frontal grey matter volume. This finding is of interest as it totally replicates their earlier seminal study. Findings of their recent studies would also appear to support the use of rTMS in people with vascular depression. Of course, several questions remain. What is the appropriate clinical phenotype (assuming there is one) of $\mathrm{VaD}$ to select subjects for treatment? On what other characteristics should subjects be selected for treatment? Why is response greater in those with $\mathrm{VaD}$ as opposed to nonvascular cases, especially since structural brain changes like frontal atrophy (which are more common in those with VaD) actually appear to be associated with poorer response to rTMS? Will effects of rTMS persist in the longer term and how should rTMS be used in conjunction with other therapies for depression, whether pharmacological or psychological? What is the dose response for rTMS? The fact that these exciting findings raise a number of other important scientific questions is 
a testament to the pioneering TMS work of Manes, Jorge, Robinson and colleagues. The authors are to be congratulated for following their earlier paper with larger, better designed, controlled studies and for tackling the subject of appropriate treatments for $\mathrm{VaD}$. They conclude in their reflection that "the available evidence clearly supports the need of further research in this field". I fully agree and, like others, I await the results of such research with great interest.

JOHN T. O'BRIEN

Deputy Editor International Psychogeriatrics Newcastle University, Newcastle, U.K.

Email: J.T.O’Brien@ncl.ac.uk

\section{References}

Alexopoulos, G. S., Meyers, B. S., Young, R. C., Campbell, S., Silbersweig, D. and Charleson, $M$. (1997). Vascular depression hypothesis. Archives of General Psychiatry, 54, 915-922.

Alexopoulos, G. S., Kiosses, D. N., Choi, S. J., Murphy, C. F. and Lim, K. O. (2002). Frontal white matter microstructure and treatment response of late-life depression: a preliminary study. American fournal of Psychiatry, 159, 1929-1932.

Almeida, O. P. (2008). Vascular depression: myth or reality? International Psychogeriatrics, 20, 645-652.

Almeida, O. P., Burton, E. J., Ferrier, N., McKeith, I. G. and O'Brien, J. T. (2003). Depression with late onset is associated with right frontal lobe atrophy. Psychological Medicine, 33, 675-681.

Andreescu, C., Chang, C.-C. H., Mulsant, B. H. and Ganguli, M. (2008). Twelve-year depressive symptom trajectories and their predictors in a community sample of older adults. International Psychogeriatrics, 20, 221-236.

Butters, M. A. et al. (2000). Changes in cognitive functioning following treatment of late-life depression. American fournal of Psychiatry, 157, 1949-1954.

Butters, M. A. et al. (2004). The nature and determinants of neuropsychological functioning in late-life depression. Archives of General Psychiatry, 61, 587-595.

Charney, D. S. et al. (2003). Depression and Bipolar Support Alliance: consensus statement on the unmet needs in diagnosis and treatment of mood disorders in late life. Archives of General Psychiatry, 60, 664-672.

Chuan, S. K., Kumar, R., Matthew, N., Heok, K. E. and Pin, N. T. (2008). Subsyndromal depression in old age: clinical significance and impact in a multi-ethnic community sample of elderly Singaporeans. International Psychogeriatrics, 20, 188-200.

Couturier, J. L. (2005). Efficacy of rapid-rate repetitive transcranial magnetic stimulation in the treatment of depression: a systematic review and meta-analysis. Fournal of Psychiatry and Neurosciences, 30, 83-90.

Ephraim, E. and Prettyman, R. (2009). Attitudes of old age psychiatrists in England and Wales to the use of mood stabilizer drugs. International Psychogeriatrics, 21, 576-580.
Fabre, I. et al. (2004). Antidepressant efficacy and cognitive effects of repetitive transcranial magnetic stimulation in vascular depression: an open trial. International fournal of Geriatric Psychiatry, 19, 833-842.

Gershon, A. A., Dannon, P. N. and Grunhaus, L. (2003). Transcranial magnetic stimulation in the treatment of depression. American fournal of Psychiatry, 160, 835845.

Grunhaus, L. et al. (2003). A randomized controlled comparison of electroconvulsive therapy and repetitive transcranial magnetic stimulation in severe and resistant nonpsychotic major depression. Biological Psychiatry, 53, 324-331.

Hickie, I., Scott, E., Mitchell, P., Wilhelm, K., Austin, M. P. and Bennett, B. (1995). Subcortical hyperintensities on magnetic resonance imaging: clinical correlates and prognostic significance in patients with severe depression. Biological Psychiatry, 37, 151-160.

Janicak, P. G. et al. (2002). Repetitive transcranial magnetic stimulation versus electroconvulsive therapy for major depression: preliminary results of a randomized trial. Biological Psychiatry, 51, 659-667.

Jorge, R. E., Moser, D. J., Acion, L. and Robinson, R. G. (2008). Treatment of vascular depression using repetitive transcranial magnetic stimulation. Archives of General Psychiatry, 65, 268-276.

Krishnan, K. R., Hays, J. C. and Blazer, D. G. (1997). MRI-defined vascular depression. American fournal of Psychiatry, 154, 497-501.

Krishnan, K. R. et al. (2004). Clinical characteristics of magnetic resonance imaging-defined subcortical ischemic depression. Biological Psychiatry, 55, 390-397.

Lebowitz, B. D. et al. (1997). Diagnosis and treatment of depression in late life: consensus statement update. $\mathcal{F} A M A$, 278, 1186-1190.

Lesser, I. M., Boone, K. B., Mehringer, C. M., Wohl, M. A., Miller, B. L. and Berman, N. G. (1996). Cognition and white matter hyperintensities in older depressed patients. American fournal of Psychiatry, 153, 1280-1287.

Levy, R. M., Steffens, D. C., McQuoid, D. R., Provenzale, J. M., MacFall, J. R., and Krishnan, K. R. R. (2003). MRI lesion severity and mortality in geriatric depression. American fournal of Geriatric Psychiatry, 11, 678-682.

Loo, C. K. and Mitchell, P. B. (2005). A review of the efficacy of transcranial magnetic stimulation (TMS) treatment for depression, and current and future strategies to optimize efficacy. Fournal of Affective Disorders, 88, 255-267.

Lyness, J. M., Chapman, B. P., McGriff, J., Drayer, R. and Duberstein, P. R. (2009). One-year outcomes of minor and subsyndromal depression in older primary care patients. International Psychogeriatrics, 21, 60-68.

Manes, F., Jorge, R., Morcuende, M., Yamada, T., Paradiso, S. and Robinson, R. G. (2001). A controlled study of repetitive transcranial magnetic stimulation as a treatment of depression in the elderly. International Psychogeriatrics, 13, 225-231.

Mosimann, U. P. et al. (2004). Repetitive transcranial magnetic stimulation: a putative add-on treatment for major depression in elderly patients. Psychiatry Research, 126, 123-133. 
Mulsant, B. H. and Ganguli, M. (1999). Epidemiology and diagnosis of depression in late life. Fournal of Clinical Psychiatry, 60 (Suppl. 20), 9-15.

Nebes, R. D. et al. (2001). Dual-task performance in depressed geriatric patients. Psychiatry Research, 102, 139-151.

Nebes, R. D. et al. (2002). Longitudinal increase in the volume of white matter hyperintensities in late-onset depression. International fournal of Geriatric Psychiatry, 17, 526-530.

O'Brien, J., Ames, D., Chiu, E., Schweitzer, I., Desmond, P. and Tress, B. (1998). Severe deep white matter lesions and outcome in elderly patients with major depressive disorder: follow-up study. BMF, 317, 982-984.

O'Reardon, J. P. et al. (2007). Efficacy and safety of transcranial magnetic stimulation in the acute treatment of major depression: a multisite randomized controlled trial. Biological Psychiatry, 62, 1208-1216.

Raskin, J., Xu, J. Y. and Kajdasz, D. K. (2008). Time to response for duloxetine $60 \mathrm{mg}$ once daily versus placebo in elderly patients with major depressive disorder. International Psychogeriatrics, 20, 309-327.

Roose, S. P. and Schatzberg, A. F. (2005). The efficacy of antidepressants in the treatment of late-life depression. Fournal of Clinical Psychopharmacology, 25, S1-S7.

Roose, S. P. et al. (2004). Antidepressant pharmacotherapy in the treatment of depression in the very old: a randomized, placebo-controlled trial. American fournal of Psychiatry, 161, 2050-2059.

Salloway, S. et al. (1996). MRI and neuropsychological differences in early- and late-life-onset geriatric depression. Neurology, 46, 1567-1574.
Salloway, S. et al. (2002). MRI subcortical hyperintensities in old and very old depressed outpatients: the important role of age in late-life depression. Fournal of the Neurological Sciences, 203, 227-233.

Schatzberg, A. and Roose, S. (2006). A double-blind, placebo-controlled study of venlafaxine and fluoxetine in geriatric outpatients with major depression. American Fournal of Geriatric Psychiatry, 14, 361-370.

Simpson, S. W., Jackson, A., Baldwin, R. C. and Burns, A. (1997). IPA/ Bayer Research Awards in Psychogeriatrics. Subcortical hyperintensities in late-life depression: acute response to treatment and neuropsychological impairment. International Psychogeriatrics, 9, 257-275

Simpson, S., Baldwin, R. C., Jackson, A., Burns, A. and Thomas, P. (2000). Is the clinical expression of late-life depression influenced by brain changes? MRI subcortical neuroanatomical correlates of depressive symptoms. International Psychogeriatrics, 12, 425434.

Sneed, J. R., Roose, S. P., Keilp, J. G., Krishnan, K. R., Alexopoulos, G. S. and Sackeim, H. A. (2007). Response inhibition predicts poor antidepressant treatment response in very old depressed patients. American fournal of Geriatric Psychiatry, 15, 553-563.

Steffens, D. C., Bosworth, H. B., Provenzale, J. M. and MacFall, J. R. (2002). Subcortical white matter lesions and functional impairment in geriatric depression. Depression and Anxiety, 15, 23-28.

Taylor, W. D. et al. (2003). White matter hyperintensity progression and late-life depression outcomes. Archives of General Psychiatry, 60, 1090-1096. 